\title{
Space-based detectors
}

\author{
A. Sesana - W. J. Weber • C. J. Killow - M. Perreur-Lloyd • D. I. Robertson • \\ H. Ward - E. D. Fitzsimons - J. Bryant - A. M. Cruise - G. Dixon • \\ D. Hoyland • D. Smith • J. Bogenstahl • P. W. McNamara - R. Gerndt • \\ R. Flatscher • G. Hechenblaikner - M. Hewitson • O. Gerberding • S. Barke • \\ N. Brause • I. Bykov • K. Danzmann - A. Enggaard • A. Gianolio • \\ T. Vendt Hansen · G. Heinzel • A. Hornstrup • O. Jennrich • J. Kullmann • \\ S. Møller-Pedersen - T. Rasmussen - J. Reiche - Z. Sodnik • M. Suess • \\ M. Armano - T. Sumner - P. L. Bender - T. Akutsu • \\ DECIGO working group • B. S. Sathyaprakash
}

Received: 11 May 2014 / Accepted: 16 August 2014 / Published online: 13 November 2014 (C) Springer Science+Business Media New York 2014

\begin{abstract}
The parallel session C5 on Space-Based Detectors gave a broad overview over the planned space missions related to gravitational wave detection. Overviews of the revolutionary science to be expected from LISA was given by Alberto Sesana and Sasha Buchman. The launch of LISA Pathfinder (LPF) is planned for 2015. This mission and its payload "LISA Technology Package" will demonstrate key technolo-
\end{abstract}

This article belongs to the Topical Collection: The First Century of General Relativity: GR20/Amaldi10. Guest Editors: Jerzy Lewandowski, Bala Iyer, Sheila Rowan.

A. Sesana

Max-Planck-Institut für Gravitationsphysik, Albert Einstein Institut, Am Mühlenberg 1, 14476 Golm, Germany

\section{W. J. Weber}

Department of Physics, University of Trento, and Trento Institute for Fundamental Physics and Applications / INFN, 38123, Povo TN, Italy

C. J. Killow · M. Perreur-Lloyd · D. I. Robertson · H. Ward

SUPA, Institute for Gravitational Research, School of Physics and Astronomy, Glasgow University, Glasgow, G12 8QQ, UK

E. D. Fitzsimons · R. Gerndt · R. Flatscher · G. Hechenblaikner Airbus Defence and Space GmbH, Claude-Dornier-Strasse, 88090 Immenstaad, Germany

J. Bryant · A. M. Cruise · G. Dixon · D. Hoyland · D. Smith School of Physics and Astronomy, University of Birmingham, Edgbaston, Birmingham B15 2TT, UK

J. Bogenstahl · O. Gerberding · S. Barke · N. Brause $\cdot$ I. Bykov · K. Danzmann · G. Heinzel · J. Kullmann · J. Reiche Institute for Gravitational Physics, Leibniz Universität Hannover, Callinstrasse 38, 30167 Hannover, Germany 
gies for LISA. In this context, reference masses in free fall for LISA, and gravitational physics in general, was described by William Weber, laser interferometry at the picometre level and the optical bench of LPF was presented by Christian Killow and the performance of the LPF optical metrology system by Paul McNamara. While LPF will not yet be sensitive to gravitational waves, it may nevertheless be used to explore fundamental physics questions, which was discussed by Michele Armano. Some parts of the LISA technology that are not going to be demonstrated by LPF, but under intensive development at the moment, were presented by Oliver Jennrich and Oliver Gerberding. Looking into the future, Japan is studying the design of a mid-frequency detector called DECIGO, which was discussed by Tomotada Akutsu. Using atom interferometry for gravitational wave detection has also been recently proposed, and it was critically reviewed by Peter Bender. In the nearer future, the launch of GRACE Follow-On (for Earth gravity observation) is scheduled for 2017, and it will include a Laser Ranging Interferometer as technology demonstrator. This will be the first interspacecraft laser interferometer and has many aspects in common with the LISA long arm, as discussed by Andrew Sutton.

Keywords LISA Pathfinder · Optical bench Interferometer - Spaceborne precision metrology $\cdot$ Interferometry $\cdot$ Phasemeter $\cdot$ Gravitational waves

P. W. McNamara · O. Jennrich · A. Gianolio · Z. Sodnik · M. Suess

European Space Research and Technology Centre, European Space Agency, Keplerlaan 1, 2200 AG, Noordwijk, The Netherlands

\section{Hewitson}

Max Planck Institute for Gravitational Physics, Albert Einstein Institute, Callinstrasse 38

30167 Hannover, Germany

T. Vendt Hansen · T. Rasmussen · A. Enggaard

Axcon ApS, Diplomvej 381, 2800 Kgs. Lyngby, Denmark

A. Hornstrup · S. Møller-Pedersen

DTU Space, National Space Institute, The Technical University of Denmark, Elektrovej 327 , 2800 Kgs. Lyngby, Denmark

\section{Armano}

European Space Astronomy Centre, European Space Agency, Madrid, Spain

T. Sumner

Imperial College London, London, UK

P. L. Bender

Boulder, CO, USA

\section{T. Akutsu}

National Astronomical Observatory of Japan, 2-21-1 Osawa, Mitaka, Tokyo 181-8588, Japan

\section{B. S. Sathyaprakash $(\varangle)$}

School of Physics and Astronomy, Cardiff University, Cardiff, UK

email: B.Sathyaprakash@astro.cf.ac.uk 


\section{The Gravitational Universe (A. Sesana)}

The Gravitational Universe [1] is a white paper describing the science case of the gravitational wave $(\mathrm{GW})$ space based interferometer eLISA, which has now been selected for the L3 launch slot by the European Space Agency (ESA). eLISA is particularly sensitive to GWs in the $\mathrm{mHz}$ frequency band, where a rich variety of sources is expected. The enormous scientific payouts related to space based interferometers are far too many to be covered in this short contribution. The reader can only get a sense of it from the following discussion, a complete overview can be found in [1].

Space based interferometers are the only detectors for which we have 'verification sources' [2]. These are the known ultra-compact binaries emitting in the $\mathrm{mHz}$ range with high signal-to-noise ratio (SNR), and will serve as verification sources for the calibration of eLISA. Assuming the eLISA design given in [1], eight such binaries are known to date. Moreover eLISA will be sensitive to the whole population of wide double white dwarf systems $\left(\sim 10^{8}\right.$ sources) populating the Galaxy. Most of those add-up to the overall confusion foreground [3,4], but about 3,000 of them should be individually resolved. Although rarer, eLISA will also detect a substantial population of short-period neutron star and black hole binaries, determining their local merger rate.

One of the most promising science goals of eLISA is the direct detection of massive black holes (MBHs), which are key building blocks in the cosmological structure formation [5,6]. Their seeding in the high redshift Universe and their early accretion are still poorly understood, and eLISA will provide unique high redshift measurements to shed light on their cosmic history. In fact, hierarchical models of $\mathrm{MBH}$ assembly predict that 10-100 MBH mergers per year occur in the Universe (see, e.g., [7]), the bulk of which involves systems with $10^{4} \mathrm{M}_{\odot}-10^{6} \mathrm{M}_{\odot}$ MBHs at $3<z<10$, falling in the sweet-spot of the eLISA sensitivity curve. eLISA will observe those binaries out to a redshift as remote as $z \sim 20$ with a SNR $>10$ (see Fig. 1). Redshifted masses will be measured to an unprecedented accuracy, up to the $0.1-1 \%$ level, whereas absolute errors in the spin determination are expected to be in the range 0.01-0.1, allowing us to reconstruct their cosmic evolution [7].

On smaller scales, eLISA will also offer the deepest view of galactic nuclei, exploring the dynamics of stars in the space-time of a Kerr BH, by capturing the GWs emitted by stellar BHs orbiting the central $\mathrm{MBH}$ (so called EMRI sources). A $10 \mathrm{M}_{\odot}$ BH inspiralling into a $10^{4} \mathrm{M}_{\odot}-10^{6} \mathrm{M}_{\odot} \mathrm{MBH}$ can be detected up to $z \simeq 0.5-0.7$ [1], allowing to explore a volume of several tens of $\mathrm{Gpc}^{3}$ and to discover MBHs in dwarf galaxies that are still elusive to electromagnetic observations. Detailed measurement of the $\mathrm{MBH}$ mass and spin (up to one part in a thousand [8]) will enable us to characterize the population of nuclear MBHs in an interval of masses where electromagnetic observations are poor, incomplete or even missing [10], providing precise measurements of their spins.

High SNR measurement of the GW waveforms will allow to test GR in the highly dynamical strong field regime of MBHs, including no-hair theorem tests, constrains on a putative graviton mass and on alternative theory of gravity in general. Making eLISA a unique probe also in the fundamental physics prospective. 


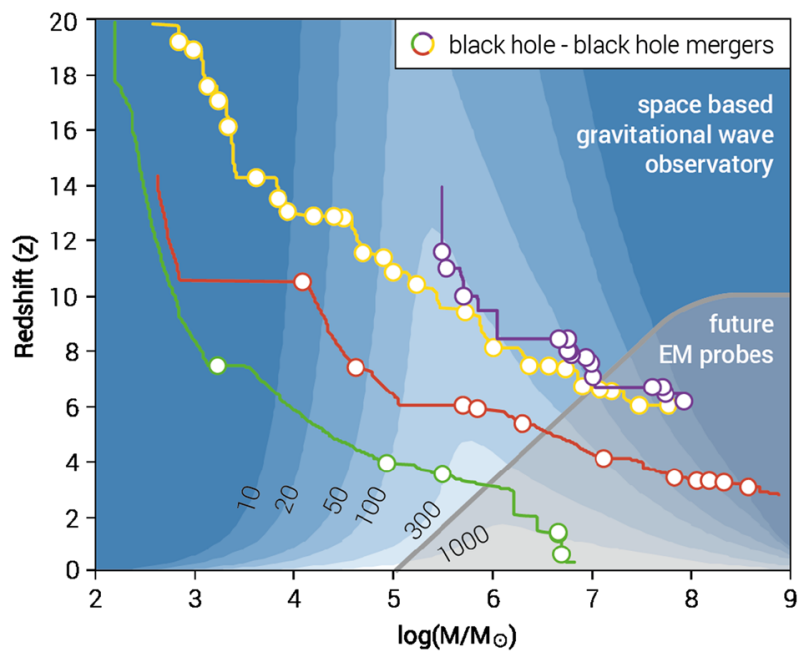

Fig. 1 Constant-contour levels of the sky and polarization angle averaged SNR for eLISA, for equal mass non-spinning binaries as a function of their total rest frame mass, $M$, and cosmological redshift, $z$. The tracks represent the mass-redshift evolution of selected supermassive BHs: two possible evolutionary paths for a $\mathrm{BH}$ powering a $z \approx 6$ quasar (starting from a massive seed, blue curve, or from a Pop III seed from a collapsed metal-free star, yellow curve); a typical $10^{9} \mathrm{M}_{\odot} \mathrm{BH}$ in a giant elliptical galaxy (red curve); and a Milky Way-like BH (green curve). Circles mark BH-BH mergers occurring along the way [9]. The grey transparent area in the bottom right corner roughly identifies the parameter space for which MBHs might power phenomena that will likely be observable by future electromagnetic probes (color figure online)

\section{Geodesic reference masses for LISA Pathfinder, eLISA, and beyond (W.J. Weber)}

Free-falling geodesic reference test masses are the core of many experiments in gravitation and in particular for gravitational wave observation. LISA Pathfinder (LPF) plans to demonstrate the possibility of measuring a differential acceleration between macroscopic test masses to within roughly $10^{-14} \mathrm{~m} / \mathrm{s}^{2} / \mathrm{Hz}^{1 / 2}$ - a femto-g/ $\mathrm{Hz}^{1 / 2}$-at $\mathrm{mHz}$ frequencies. This ambitious performance goal places high demands on the system, known as the Gravitational Reference Sensor, in charge of sensing test mass displacement and establishing the environment compatible with the desired free-fall purity.

The LPF performance goal represents a roughly five order of magnitude improvement on the best performance demonstrated to date in this frequency band by the recently completed geodesy mission GOCE [11]. The LPF measurement [12] is conceptually equivalent to one of GOCE's nine gravitational gradient components, measuring $\frac{\partial g_{x}}{\partial x}$ in the differential acceleration of two test-masses separated by a $38 \mathrm{~cm}$ baseline. To achieve this large improvement upon the current state of the art, the LPF GRS $[13,14]$ employs a number of new design features:

- Spacecraft drag-free control in a benign environment of the first Lagrange point (L1), where the required test mass forcing is reduced from the $\mu \mathrm{m} / \mathrm{s}^{2}$ level of low earth orbit to sub-nm/s $\mathrm{s}^{2}$, limited by the imperfect balancing of the spacecraft mass distribution. This still remains a performance-limiting issue for LPF, but not for 
eLISA, where the test masses can be left force-free along the critical interferometry measurement axes.

- No mechanical contact couples the test mass to the spacecraft, with the discharge wire used in geodesy accelerometers removed in LPF to leave the TM truly floating and without the thermodynamic noise associated with mechanical damping in the wire [15].

- Heavy mass and big gaps. LPF uses a high-density, low-magnetic Au-Pt cubic ( $46 \mathrm{~mm}$ and $2 \mathrm{~kg}$ ) test mass, surrounded by several $\mathrm{mm}$ vacuum gaps. These mass and gap sizes are both roughly an order of magnitude increase from GOCE, and aim at reducing the acceleration noise from stray forces acting on the test mass surface, particularly from low frequency electrostatics [16] and Brownian force noise from gas damping [17]. Current torsion pendulum surface force noise measurements with prototype LPF GRS hardware place upper limits, of roughly $20 \mathrm{fm} / \mathrm{s}^{2} / \mathrm{Hz}^{1 / 2}$ around $3 \mathrm{mHz}$, already nearly compatible with the LPF flight goals [18].

An updated analysis of the LPF noise budget from 2011 [12], based on experimental studies of the various acceleration noise sources with flight and flight-prototype hardware shows that LPF should demonstrate the required eLISA performance of a single test mass acceleration noise of $3 \mathrm{fm} / \mathrm{s}^{2} / \mathrm{Hz}^{1 / 2}$ at frequencies above $1 \mathrm{mHz}$. Additionally, the interferometry readout sensitivity needed to achieve the acceleration measurement, better than roughly $10 \mathrm{pm} / \mathrm{Hz}^{1 / 2}$, has been demonstrated in lab with the actual flight optical bench and phase readout (see C. Killow in this session).

In addition to establishing a benchmark for GRS performance for eLISA, LPF will help construct a physical model for all sources limiting the precision with which macroscopic test masses can be used as geodesic references, across a wide range of frequencies, operating conditions, and performance levels, with possible impact on future geodesy missions (see for instance [19]) and fundamental tests of general relativity (see for instance [20]).

\section{Optical bench interferometer for LISA Pathfinder (C.J. Killow)}

The LISA Pathfinder Optical Bench Interferometer (OBI) provides the precision sensing of the critical degrees of freedom of two inertially free test masses. The required test mass to optical bench measurement sensitivity is $10 \mathrm{pm}$ and $20 \mathrm{nrad}$. The approach taken has been to build a set of intrinsically stable Mach-Zehnder heterodyne interferometers whose laser beams travel different paths, with all components rigidly mounted on the OBI baseplate, a $\sim 20 \mathrm{~cm}$ square and few $\mathrm{cm}$ thick slab of low expansion material that is mounted in the region between the two test masses. The monitoring of changes in the relative phases of the heterodyne signals at interferometer outputs yields information on optical path variations. For example the heterodyne signal from an interferometer that has included a reflection from a test mass can be compared to that from a reference interferometer that has involved exclusively fixed paths to monitor test mass motion compared to the stable optical bench. The details of the design, construction and testing of the OBI can be found in [21].

In order to produce an optical assembly that simultaneously possesses the required optical alignment, stability, and mechanical robustness, the hydroxide-catalysis bond- 
ing technique was adapted for precision component alignment [22]. This necessitated a parallel development of a laser beam vector measurement device [23] to readout beam positions, and to act as an alignment target. The ability to control laser beam pointing was vital for centering beams on the nominal test masses within the required $\pm 25 \mu \mathrm{m}-$ a target that was comfortably met at both test masses.

A development of note for the OBI were the novel glass fibre injectors that are used to bring light from the laser and modulation system to the optical bench. These have a demonstrated beam pointing stability of no more than $3.4 \mu \mathrm{rad} /{ }^{\circ} \mathrm{C}$. The intensity beam profiles of the two fibre injectors were matched for the optical path difference on the OBI of $384 \mathrm{~mm}$, resulting in wavefront matching at the main beam combining beamsplitters of better than $\lambda / 20$ and enabling contrast values of $\geq 90 \%$ for all four interferometers.

The flight OBI underwent environmental testing with no degradation in performance and was successfully delivered to Astrium $\mathrm{GmbH}$ for further integration into the LISA Pathfinder Core Assembly. The techniques and capabilities developed for the OBI are now being applied to the more complex optical sensing requirements of a full space-borne gravitational wave detector mission.

Acknowledgements The authors would like thank the international LISA Pathfinder community for many helpful contributions towards this work. We acknowledge financial support from SUPA, University of Glasgow, STFC, UKSA and ESA.

\section{Performance testing of the LPF optical metrology system (P.W. McNamara)}

LISA Pathfinder [24] is an ESA mission designed to test the concept of low frequency gravitational wave detection metrology in a space environment. Specifically, LPF will demonstrate that a macroscopic test mass can be put into pure free-fall to within a factor of ten of the performance required by the proposed eLISA mission [25]. To achieve this goal, LPF will demonstrate a drag-free control system consisting of: inertial sensors, high precision optical metrology, drag free control algorithms, and a micro-Newton propulsion system.

LISA Pathfinder is now nearing launch, with most of the flight hardware having been delivered and integrated to the spacecraft. This has allowed the flight system to start the necessary environmental tests required prior to launch. One such test, the OnStation Thermal Test (OSTT) provided an opportunity to test the Optical Metrology System (OMS) in a flight-like environment - the first such test of its kind.

The OMS consists of several units, namely: the reference laser unit, the laser modulator, the optical bench, the phasemeter, and the payload computer. As the OSTT took place in a thermal vacuum chamber in a 1-g environment, the free-floating test masses of the inertial sensors could not be realised, and therefore were replaced by PZT-mounted mirrors. Apart from these mirrors, all other units consist of the flight hardware.

The OSTT is primarily a thermal test of the spacecraft and payload, and is aimed at validating the thermal model of the system. As such, the tests are performed at both extremes of the expected thermal environment, thereby providing the opportunity to test the OMS also at both extremes. In all, several performance runs were made over 

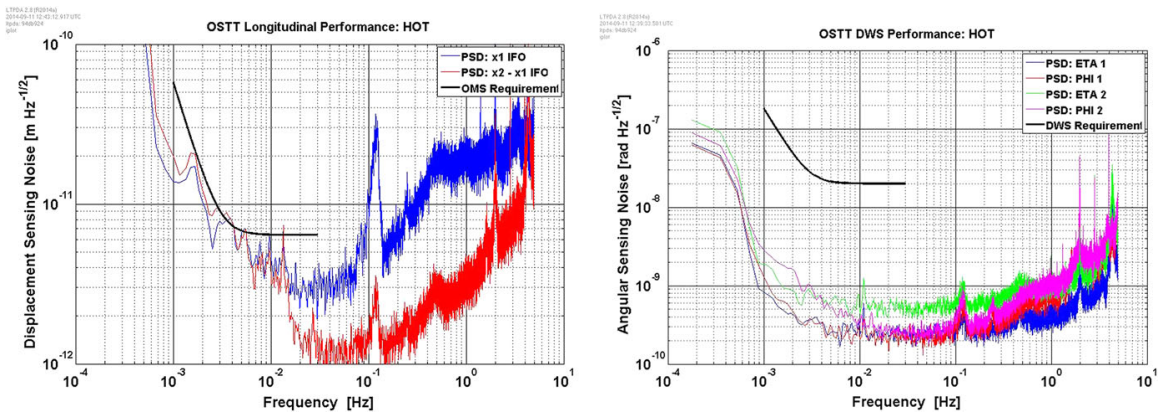

Fig. 2 Left Spectra of the longitudinal performance of the LPF Optical Metrology System: Blue $=\mathrm{x}_{1}$ interferometer, Red $=\mathrm{x}_{2}-\mathrm{x}_{1}$ (differential) interferometer. Right Spectra of the angular sensing noise of the LPF OMS. The solid black line on both charts shows the optical sensing noise requirement (color figure online)

the span of approximately three weeks at both the hot $\left(\sim 28^{\circ} \mathrm{C}\right)$ and cold $\left(\sim 12^{\circ} \mathrm{C}\right)$ temperature bounds.

The requirements levied on the optical metrology system stem from the eLISA local interferometer sensing noise requirements [25], namely a longitudinal sensing noise of $<6.4 \mathrm{pm} / \sqrt{\mathrm{Hz}}$ rising as $f^{2}$ below $3 \mathrm{mHz}$, and an angular sensing noise (utilising differential wavefront sensing) of $<20 \mathrm{nrad} / \sqrt{\mathrm{Hz}}$ rising as $f^{2}$ below $3 \mathrm{mHz}$. All performance runs demonstrated that the OMS performance meets, and significantly exceeds, the imposed requirements. Figure 2 shows both the measured longitudinal (left) and angular (right) sensing noise spectra.

As can be seen, the performance of the OMS exceeds the requirements, with the $\mathrm{x}_{2}-\mathrm{x}_{1}$ interferometer reaching sensitivity of $<2 \mathrm{pm} / \sqrt{\mathrm{Hz}}$ above $20 \mathrm{mHz}$, and the angular sensing noise exceeding the requirements by $\sim 2$ orders of magnitude.

Acknowledgements The OSTT took place in the large space simulator at the IABG, Germany, under the authority of Astrium Ltd, the LPF prime industrial contractor. The OMS test campaign was performed under the responsibility of Astrium GmbH, the LISA Technology Package Architect. The author wishes to thank the full LISA Pathfinder team, including both industry and university teams, who have designed, built and tested the LPF hardware.

\section{Testing the breadboard model of the LISA phasemeter (O. Gerberding)}

Future space-borne gravitational wave detectors, like the Laser Interferometer Space Antenna (LISA) or its variant evolved LISA (eLISA) [1], utilize MHz heterodyne interferometry as prime measurement technology. Therefore they require a metrology system, or phasemeter [26], that performs the phase measurement of the interferometric beat notes, between 5 and $25 \mathrm{MHz}$, with $\mu \mathrm{rad} / \sqrt{\mathrm{Hz}}$ performance at frequencies between $0.1 \mathrm{mHz}$ and $1 \mathrm{~Hz}$. The phasemeter is also required to include various additional functionalities, including the capability to perform inter-satellite ranging and communication [28,29], laser offset-phase locking [30], support for the dragfree and attitude control system (DFACS) and a subsystem providing a system clock, 
ultra-stable pilot tone, and $\mathrm{GHz}$ tones for clock tone transfer with high relative phase stabilities [31]. This subsystem is also called frequency distribution system (FDS).

An elegant breadboard model (EBB) of such a phasemeter system has been developed in the frame of an ESA technology development activity by a consortium composed of the National Space Institute of the Danish Technical University, the Danish industry partner Axcon ApS in Copenhagen, and the Albert Einstein Institute in Hannover [27]. The EBB has been constructed and it is currently beeing tested for all desired functionalities in an extensive test campaign. A dedicated digital signal simulator has also been constructed to generate analogue LISA-like signals for the performance tests. First measurements revealed that the system is able to achieve $\mu \mathrm{rad} / \sqrt{\mathrm{Hz}}$ performance, if critical parts are thermally isolated.

An optical set-up for performing integrated tests of the EBB is also under development. This set-up will allow non-linearity tests of the phasemeter [32] to be performed, by generating three interferometric beat notes with phases that can be combined to zero. These test will make it possible to investigate noise sources that are not detectable with the currently used zero-measurement scheme, where the same signal is split and measured by different phasemeter channels. The use of independent phasemeters, with separate clocks, will also allow the integration of the clock tone transfer into the measurement. This will require a well performing clock noise correction scheme to achieve the final performance. Therefore the complete LISA metrology system will be tested in a single set-up.

Acknowledgements This work was funded by the European Space Agency (ESA) as part of the technology development for LISA. The authors gratefully acknowledge the support by the International Max-Planck Research School for Gravitational Wave Astronomy (IMPRS-GW), by QUEST (Centre for Quantum Engineering and SpaceTime Research) and by Deutsches Zentrum für Luft-und Raumfahrt (DLR) (reference 50 OQ 0601).

\section{LISA Pathfinder and alternative theories of gravity (M. Armano)}

The LISA Pathfinder (LPF) mission, to be flown by ESA, will carry a gravity gradiometer of unprecedented sensitivity $\left(\sim 7.5 \times 10^{-14} \mathrm{~ms}^{-2} / \mathrm{m} / \sqrt{\mathrm{Hz}}\right)$. After the LPF mission it is proposed to change the orbit to pass through the Sun-Earth saddle point (Fig. 3, left panel, from S. Kemble, Astrium UK) to measure gravity gradients in this extremely weak field environment, providing additional information to planetary ephemerides (Fig. 3, right panel, from A. Mozaffari, Imperial College London). A null result will conclusively test some theories [33,34]; a positive result would have great fundamental implications.

\section{Issues for suggested single photon recoil atom interferometry gravitational wave measurements in space (P.L. Bender)}

A paper published recently by Graham et al. [35] suggested the use of single photon atomic transitions for possible future gravitational wave observations in space 

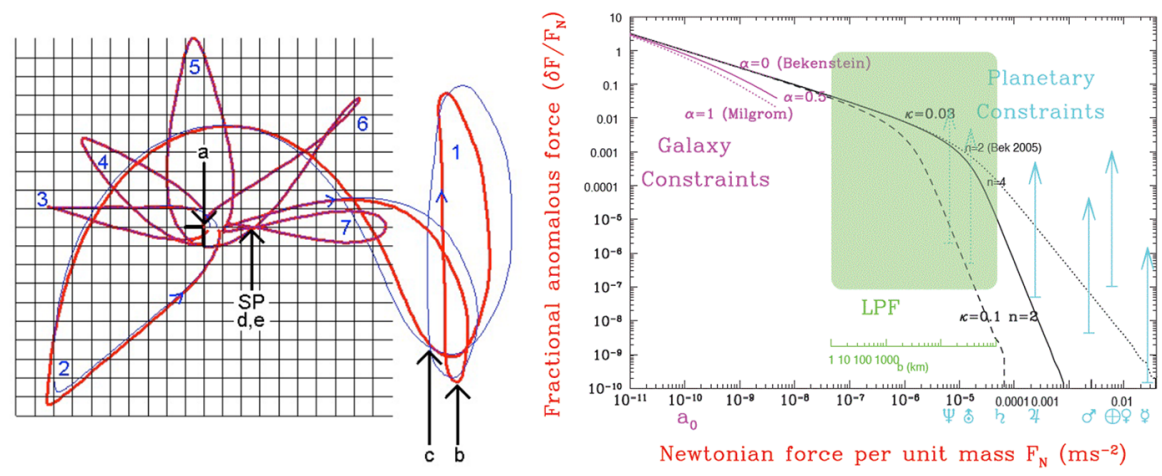

Fig. 3 Possible LPF orbits close to Sun-Earth saddle point (left) and residual accelerations which LPF can maeasure near such orbits (right)

based on atom interferometry. Instead of two photon Raman transitions, single photon transitions on highly forbidden optical lines would be employed. In the example given, the transition used is the $698 \mathrm{~nm}$ optical clock line in $87 \mathrm{Sr}$, and the gravitational wave sensitivity goal at $0.01 \mathrm{~Hz}$ is $10^{-20} \mathrm{~Hz}^{-1 / 2}$. Also assumed are the following: a $1,000 \mathrm{~km}$ baseline between two atom interferometers illuminated by the same lasers pulses; large momentum transfer sequences of laser pulses producing 2,400 optical transitions in each atom; and an observation time of $100 \mathrm{~s}$.

In order to understand the requirements for a space mission consistent with the example given, assumptions about the laser power, the atom cloud temperature, and the methods for reading out the atom wavefunction phase differences in the two interferometers need to be made. Graham et al. say in a footnote in [35] that many trades are possible, but a specific reference case that tradeoff studies can be based on is needed in order to make the discussion useful.

One specific issue is the very high laser intensity needed at both interferometers. To achieve this, something like 1 meter diameter transmitting telescopes at each spacecraft and $30 \mathrm{~W}$ of laser power apparently are needed, and these values are adopted here for the reference case. This gives a Rabi frequency of $200 \mathrm{~Hz}$. Somewhat lower laser intensity would be possible if the $578 \mathrm{~nm}$ optical clock transition in $171 \mathrm{Yb}$ were used instead. However, there is a tradeoff involved because $171 \mathrm{Yb}$ may be more difficult to cool to extremely low temperatures than $87 \mathrm{Sr}$.

A second issue concerns the atom cloud temperature. For a Rabi frequency of $200 \mathrm{~Hz}$ and the use of square laser pulses, the temperature requirement for half the atoms to make all of the necessary transitions successfully is about $0.04 \mathrm{pK}$. Some relaxation of this requirement is possible by using different pulse designs, but it is not clear how much of a relaxation would be feasible in practice.

A more general issue is how the wavefunction phase differences would be read out for the two interferometers. It appears necessary to read out both the fringe contrast and the phase difference for each atom cloud. Sugarbaker et al. [36] have shown how this can be done either by tilting a mirror or by changing the timing of one of the pulse sequences for Raman transitions in $87 \mathrm{Rb}$. However, it does not appear that the wavefront tilting approach could be used because of the $1,000 \mathrm{~km}$ baseline involved. 
And it is not completely clear if the pulse sequence timing approach would work with single photon transitions and extremely cold atoms.

Assuming that a single cloud measurement method for both fringe phase and fringe contrast is available, it probably also would be necessary to make the measurements on two different hyperfine components of the transition on the same atom cloud. This is because of the rapid magnetic field fluctuations in space. In view of the possible complexity involved, it would be useful to have an example of how the combined fringe phase, fringe contrast, and hyperfine component measurements could be made that would permit evaluation of the number of atoms needed per cloud to reach the gravitational wave sensitivity goal.

In addition, for the suggested atomic transition and the high momentum transfer pulse sequences being considered, providing sufficiently large sunshields seems difficult. This is because of the wavefunctions for the atoms in each interferometer being split by a distance of up to about $20 \mathrm{~m}$ during the observation time.

The approach suggested by Graham et al. would indeed make possible gravitational wave measurements over a single baseline, and thus with two spacecraft rather than the three required for differential laser interferometry over three baselines, as in the proposed LISA mission [37]. This was the main point made in the paper by Graham et al. [35]. However, based on the reference mission assumptions discussed above, it unfortunately appears that the atom interferometry mission would be much more complex. Also, the scientific returns would be less because of only observing one polarization of the gravitational waves, and because of the less favorable overall sensitivity curve.

\section{DECIGO and DECIGO Pathfinder (T. Akutsu)}

In this talk we reported on the status of DECIGO (DECi-hertz Interferometer Gravitational-wave Observatory), which will be a spaceborne antenna to be launched in the 2020s, and observe gravitational-wave sources at $0.1 \mathrm{~Hz}[38,39]$. Since this spectral region is not covered by LISA or ground-based antennas, DECIGO will provide unique or complementary information to open a new window to the Universe. In the current pre-conceptual design, DECIGO comprises four interferometer units; each unit is formed by three drag-free spacecraft with 1,000-km separation, and each spacecraft holds two mirrors of about $1 \mathrm{~m}$ diameter. With $10 \mathrm{~W}$ laser sources, FabryPerot cavities are formed between the spacecraft (Fig. 4, left). The scientific objectives of DECIGO are summarized in Ref. [39]. In order to meet the scientific objectives, we start to slightly change some parameters like laser power, mirror diameters and spacecraft separation (Fig. 4, right).

We also reported on the first milestone mission, DECIGO Pathfinder (DPF), which will be a small satellite $(950 \times 950 \times 2,000 \mathrm{~mm})$, and launched to low-earth orbit in the middle of this decade. Since DECIGO is an extremely large mission, it is important to increase the technical feasibility before its launch. With the DPF, the technologies key to DECIGO will be demonstrated, and also some observation runs will be carried out. The key technologies are (1) a stabilized laser, (2) a Fabry-Perot interferometer unit, and (3) a control system of drag-free flight. For (1) and (2), a development with 

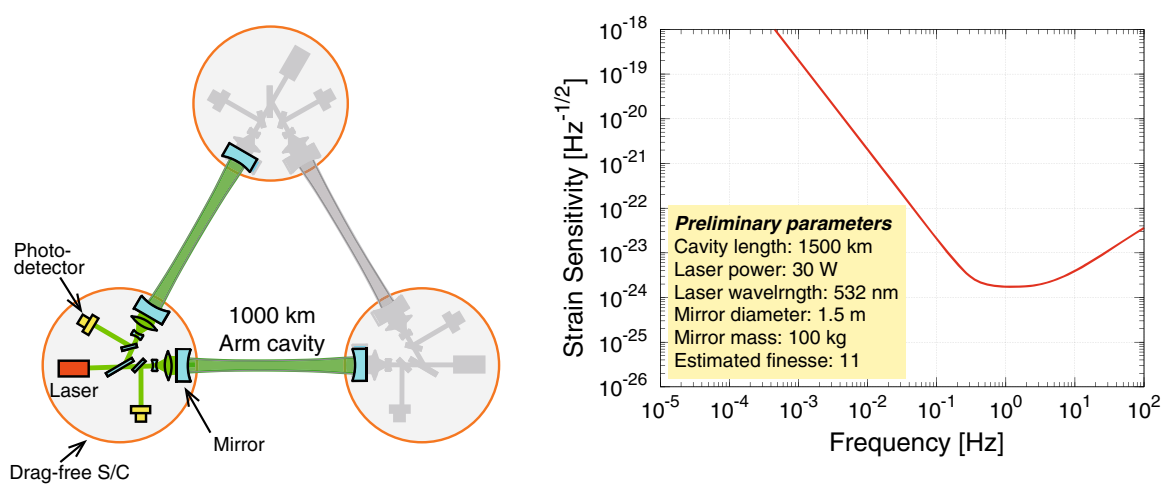

Fig. 4 Left pre-conceptual design of a single interferometer unit of DECIGO; it employs three spacecraft (S/C) in formation flight to maintain three $1,000-\mathrm{km}$ long arm cavities at their operation points. Right preliminary estimation of the goal noise level of the interferometer after some parameters are slightly tuned

(a)

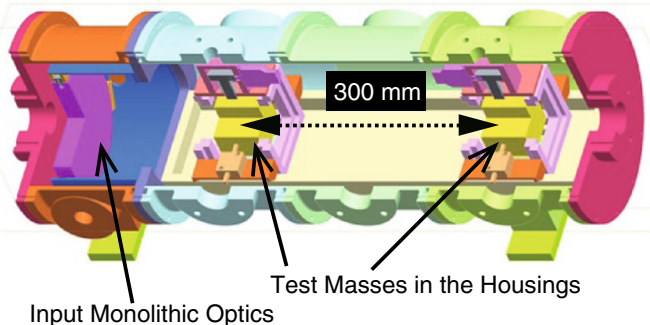

(b)

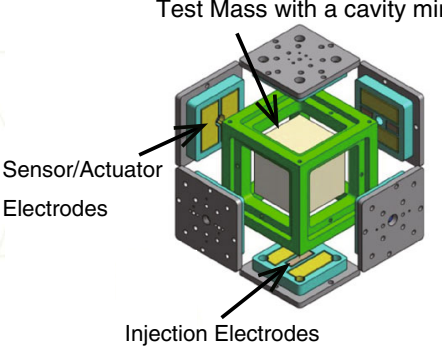

Fig. 5 Schematic view of (a) the module of the Fabry-Perot cavity interferometer on board DPF and (b) one of the test-mass housings installed in it

simple test benches has been almost finished, and a prototyping of engineering models has started. For (3), a development of the control topology has started (Fig. 5).

Acknowledgments This research was supported by the Japan Aerospace Exploration Agency (JAXA), by the Japan Society for the Promotion of Science (JSPS), Grant-in-aid for scientific research, by the Global COE Program of the graduated school of science in Kyoto University, and by the Research Center for the Early Universe (RESCEU) at the University of Tokyo.

\section{References}

1. Amaro Seoane, P., et al.: The Gravitational Universe, arXiv:1305:5720 (2013)

2. Stroeer, A., Vecchio, A.: The LISA verification binaries. Class. Quantum Gravity 23, 809 (2006)

3. Nelemans, G., Yungelson, L.R.: The gravitational wave signal from the Galactic disk population of binaries containing two compact objects. A\& A 375, 890 (2001)

4. Nissanke, S., Vallisneri, M., Nelemans, G., Prince, T.A.: Gravitational-wave emission from compact Galactic binaries. Astrophys. J. 758, 131 (2012)

5. Volonteri, M., Haardt, F., Madau, P.: The assembly and merging history of supermassive black holes in hierarchical models of galaxy formation. Astrophys. J. 582, 599 (2003) 
6. Malbon, R.K., Baugh, C.M., Frenk, C.S., Lacey, C.G.: Black hole growth in hierarchical galaxy formation. Mon. Not. R. Astron. Soc. 382, 1394 (2007)

7. Sesana, A., Gair, J., Berti, E., Volonteri, M.: Reconstructing the massive black hole cosmic history through gravitational waves. Phys. Rev. D 83, 4036 (2011)

8. Barack, L., Cutler, C.: LISA capture sources: Approximate waveforms, signal-to-noise ratios, and parameter estimation accuracy. Phys. Rev. D. 69 (2004)

9. Volonteri, M.: The early evolution of massive black holes, arXiv:0912.0525 (2009)

10. Gair, J.R., Tang, C., Volonteri, M.: LISA extreme-mass-ratio inspiral events as probes of the black hole mass function. Phys. Rev. D 81, 4014 (2010)

11. Rummel, R., Yi, W., Stummer, C.: GOCE gravitational gradiometry. J. Geodesy 85, 777 (2011)

12. Antonucci, F., et al.: From laboratory experiments to LISA Pathfinder: achieving LISA geodesic motion. Class. Quantum Gravity 28, 094002 (2011)

13. Dolesi, R., et al.: Gravitational sensor for LISA and its technology demonstration mission. Class. Quantum Gravity 20, S99 (2003)

14. Weber, W.J., et al.: Position sensors for flight testing of LISA drag-free control. SPIE Proceedings 4856, 31 (2003)

15. Willemenot, E., Touboul, P.: On-ground investigation of space accelerometers noise with an electrostatic torsion pendulum. Rev. Sci. Instr. 71, 302 (2000)

16. Antonucci, F., et al.: Interaction between stray electrostatic fields and a charged free-falling test mass. Phys. Rev. Lett. 108, 181101 (2012)

17. Cavalleri, A., et al.: Increased brownian force noise from molecular impacts in a constrained volume. Phys. Rev. Lett. 103, 140601 (2009)

18. Cavalleri, A., et al.: A new torsion pendulum for testing the limits of free-fall for LISA test masses. Class. Quantum Gravity 26, 094017 (2009)

19. Wiese, D.N., Folkner, W.M., Nerem, R.S.: Alternative mission architectures for a gravity recovery satellite mission. J. Geodesy 83, 569 (2009)

20. Ashby, N., et al.: Measurement of gravitational time delay using drag-free spacecraft and an optical clock. Proc. Int. Astr. Union Symp. 5, 414 (2009)

21. Robertson, D.I., et al.: Construction and testing of the optical bench for LISA Pathfinder. Class. Quantum Gravity 30, 085006 (2013)

22. Killow, C.J., et al.: Construction of rugged, ultrastable optical assemblies with optical component alignment at the few microradian level. Appl. Opt. 52, 177-181 (2013)

23. Fitzsimons, E.D., et al.: Precision absolute positional measurement of laser beams. Appl. Opt. 52, 2527-2530 (2013)

24. Racca, G.D., McNamara, P.W.: The LISA pathfinder mission. Sp. Sci. Rev. 151, p159-181 (2010)

25. Amaro-Seone, P., et al.: eLISA: Astrophysics and cosmology in the millihertz regime. GW Notes 6, p4-110 (2013)

26. Shaddock, D., et al.: Overview of the LISA phasemeter. AIP Conf. Proc. 873, 689-696 (2006)

27. Gerberding, O., et al.: Breadboard model of the LISA phasemeter. ASP Conference Series, 467, 271, 9th LISA Symposium (2012) arXiv.org/abs/1208.6418

28. Sutton, A.J., et al.: Improved optical ranging for space based gravitational wave detection. Class. Quantum Gravity 30, 075008 (2013)

29. Esteban, J.J., et al.: Experimental demonstration of weak-light laser ranging and data communication for LISA. Opt. Express 19, 15937 (2011)

30. McNamara, P.W.: Weak-light phase locking for LISA. Class. Quantum Gravity 22, S243 (2005)

31. Heinzel, G., et al.: Auxiliary functions of the LISA laser link: ranging, clock noise transfer and data communication. Class. Quantum Gravity 28, 094008 (2011)

32. Gerberding, O., et al.: Phasemeter core for intersatellite laser heterodyne interferometry: modelling, simulations and experiments. Class. Quantum Gravity 30, 235029 (2013)

33. Magueijo, J., Mozaffari, A.: Case for testing modified Newtonian dynamics using LISA pathfinder. Phys. Rev. D 85, 043527 (2012)

34. Sumner, T.: Science with LISA pathfinder. ASP Conf. Ser. 467, 129-140 (2013)

35. Graham, P.W., Hogan, J.M., Kasevich, M.A., Rajendran, S.: New method for gravitational wave detection with atomic sensors. Phys. Rev. Lett. 110, 171102 (2013)

36. Sugarbaker, A., Dickerson, A.M., Hogan, J.M., Johnson, D.M.S., Kasevich, M.A.: Enhanced atom interferometer readout through the application of phase shear. Phys. Rev. Lett. 111, 113002 (2013) 
37. Jennrich, O., et al.: LISA, Unveiling a hidden Universe: Assessment Study Report, ESA/SRE(2011) (2011)

38. Seto, N., Kawamura, S., Nakamura, T.: Possibility of direct measurement of the acceleration of the universe Using $0.1 \mathrm{~Hz}$ band laser interferometer gravitational wave antenna in space. Phys. Rev. Lett. 87, 221103 (2001)

39. Kawamura, S., et al.: The Japanese space gravitational wave antenna: DECIGO. Class. Quantum Gravity 28, 094011 (2011) 